\title{
ACHROMAT WITH LINEAR SPACE CHARGE FOR BUNCHED BEAMS*
}

\author{
D. Raparia, J.G. Alessi, Y. Y. Lee and W. T. Weng \\ Brookhaven National Laboratory \\ Upton, NY 11973, USA
}

\section{Abstract}

The standard definition for an achromat is a transport line having zero values for the spatial dispersion (R16) and the angular dispersion (R26). For a bunched beam with linear space charge this definition of achromaticity does not hold. The linear space charge in the presence of a bend provides coupling between (a) bunch spatial width and bunch length (R15) and (b) bunch angular spread and bunch length ( $R 25)$. Therefore, achromaticity should be redefined as a line having zero values of the spatial dispersion (R16), the angular dispersion (R26), and matrix elements $R 15$ \& $R 25$. These additional conditions $(R 15=R 25=0$ ) can be achieved, for example, with two small RF cavities at appropriate locations in the achromat, to cancel space charge effects. An example of the application of this technique to the Spallation Neutron Source (SNS) high energy beam transport line will be presented.

\section{INTRODUCTION}

A transport line is said to be achromatic if spatial \& angular widths of the beam are independent of its momentum spread. In other words, the spatial dispersion (R16) and the angular dispersion (R26) have zero values. The spatial \& angular widths are also independent of the bunch length in absence of linear space charge. The beam ellipse is upright in the $\mathrm{x}-\mathrm{z}$ plane. A property of an achromatic bend is that the total phase advance should be $n \pi(n=1,2 \ldots)$ if all dipoles in achromat bend the beam in the same direction, or $2 n \pi$ if some of the dipoles in achromat bend the beam in the opposite direction. In the presence of linear space charge the transport line no longer remains achromatic for two reasons. (1) The total phase advance is no longer $n \pi$ or $2 n \pi$ due to tune depression. The total phase advance can be changed back to $n \pi$ or $2 n \pi$ by readjusting quadrupoles in achromat. (2) When beam passes through the bend it is no longer upright in $\mathrm{x}-\mathrm{z}$ plane because space charge provides coupling between (a) bunch spatial width and bunch length (R15) and (b) bunch angular width and bunch length (R25). The beam ellipse can be made upright in $x-z$ plane by introducing two rf cavities in the achromat.

\footnotetext{
* Work performed under the auspices of U.S. Department of Energy
}

\section{ACHROMAT WITH LINEAR SPACE CHARGE FOR BUNCHED BEAM}

\subsection{Linear Space Charge for Bunched Beams}

Following the formulism of TRACE3D [1], the electric field components due to a uniformly charged ellipsoid are given by [2]

$$
\begin{gathered}
E_{x}=\frac{1}{4 \pi \epsilon_{0}} \frac{3 I \lambda}{c \gamma^{2}} \frac{(1-f)}{r_{x}\left(r_{x}+r_{y}\right) r_{z}} x, \\
E_{y}=\frac{1}{4 \pi \epsilon_{0}} \frac{3 I \lambda}{c \gamma^{2}} \frac{(1-f)}{r_{y}\left(r_{x}+r_{y}\right) r_{z}} y, \\
E_{z}=\frac{1}{4 \pi \epsilon_{0}} \frac{3 I \lambda}{c} \frac{f}{r_{x} r_{y} r_{z}} z,
\end{gathered}
$$

where $r_{x}, r_{y}$ and $r_{z}$ are the semi-axes of a ellipsoid, $I$ is the average electrical current over one RF period, $\lambda$ is the free-space wavelength of the $R F, c$ is the velocity of light, and $\epsilon_{0}$ is the permittivity of the free space. The form factor $f$ is a function of $p \equiv \frac{\gamma r_{z}}{\sqrt{r_{x} r_{y}}}$ given by

$$
f(p)= \begin{cases}\frac{1}{1-p^{2}}-\frac{p}{(1-p)^{3 / 2}} \cos ^{-1} p & \text { if } p<1 \\ \frac{p \cosh }{\left(p^{2}-1\right)^{3 / 2}}-\frac{1}{\left(p^{2}-1\right)} & \text { if } p>1 \\ \frac{1}{3} & \text { if } p=1\end{cases}
$$

where $\cosh ^{-1} p=\ln \left(p+\sqrt{p^{2}-1}\right)$.

The space charge is applied in the kick approximation as a change in the normalized momentum components as the beam traverses $\Delta s$, and is given by

$$
\Delta(\beta \gamma)_{u}=\frac{q E_{u} \Delta s}{m_{0} c^{2} \beta}
$$

where $\mathrm{u}$ represents $\mathrm{x}, \mathrm{y}$, or $\mathrm{z}$. This kick formulation is correct as long as the ellipsoid is upright in local $x-y, y-z$, and $z$-x planes. When beam traverses the bend the ellipsoid is tilted in the local $x-z$ plane. To calculate the space charge kick first one has to transform to the coordinate system in which the ellipsoid is upright. The ellipsoid is transformed back to the local coordinate system after applying the space charge kick. The transfer matrix for the space-charge kick is

$$
R_{S}=\left(\begin{array}{llllll}
1 & 0 & 0 & 0 & 0 & 0 \\
\frac{q E_{x} \Delta s}{m_{0} c^{2} \beta} & 1 & 0 & 0 & 0 & \\
0 & 0 & 1 & 0 & 0 & 0 \\
0 & 0 & \frac{q E_{y} \Delta s}{m_{0} c^{2} \beta} & 1 & 0 & 0 \\
0 & 0 & 0 & 0 & 1 & 0 \\
0 & 0 & 0 & 0 & \frac{q E_{c} \Delta s}{m_{a} c^{2} \beta} & 1
\end{array}\right) .
$$




\subsection{Coupling Between Bunch Length and Bunch Spatial \& Angular Widths}

This coupling is induced by linear space charge, which can be shown as follows. The transfer matrix of a bending magnet for bend angle $\alpha$ and length $L$ is

$$
\begin{aligned}
& R[x x]_{B}=\left(\begin{array}{ll}
\cos \left(k_{x} L\right) & \frac{1}{k_{x}} \sin \left(k_{x} L\right) \\
-k_{x} \sin \left(k_{x} L\right) & \cos \left(k_{x} L\right)
\end{array}\right) \\
& R[x z]_{B}=\left(\begin{array}{cc}
0 & \frac{h\left(1-\cos \left(k_{x} L\right)\right)}{k_{x}^{2}} \\
0 & \frac{h \sin \left(k_{x}^{2} L\right)}{k_{x}}
\end{array}\right) \\
& R[y y]_{B}=\left(\begin{array}{ll}
\cos \left(k_{y} L\right) & \frac{1}{k_{y}} \sin \left(k_{y} L\right) \\
-k_{y} \sin \left(k_{y} L\right) & \cos \left(k_{y} L\right)
\end{array}\right) \\
& R[z x]_{B}=\left(\begin{array}{ll}
\frac{-h \sin \left(k_{x} L\right)}{k_{x}} & \frac{-h\left(1-\cos \left(k_{x} L\right)\right)}{k_{x}^{2}} \\
0 & 0
\end{array}\right) \\
& R[z z]_{B}=\left(\begin{array}{ll}
1 & \frac{-1}{\rho^{2} k^{3}}\left(k_{x} L \beta^{2}-\sin \left(k_{x} L\right)\right) \\
& +\frac{L}{\gamma^{2}}\left(1-\frac{1}{\rho^{2} k_{x}^{2}}\right) \\
0 & 1
\end{array}\right) \\
& R[x y]_{B}=R[y x]_{B}=R[y z]_{B}=R[z y]_{B}=\left(\begin{array}{ll}
0 & 0 \\
0 & 0
\end{array}\right) \\
& h=\frac{1}{|\rho|} \frac{\alpha}{|\alpha|} \\
& k_{x}=\sqrt{(1-n) h^{2}} \\
& k_{y}=\sqrt{n h^{2}} \\
& L=|\rho| \alpha \\
& \rho=\frac{m_{0} c \beta \gamma}{q B_{y}} \\
& n=-\left(\frac{\rho}{B_{y}} \frac{\partial B_{y}}{\partial x}\right)_{x=0, y=0} \\
& R_{S B} 15=R_{B} 16 * R_{S} 65=\frac{h\left(1-\cos \left(k_{x} L\right)\right)}{k_{x}^{2}} \times \frac{q E_{z} L}{m_{0} c^{2} \beta} \\
& R_{S B} 25=R_{B} 26 * R_{S} 65=\frac{h \sin \left(k_{x}\right) L}{k_{x}} \times \frac{q E_{z} L}{m_{0} c^{2} \beta} .
\end{aligned}
$$

Even after applying the usual conditions for an achromat, $R 16=R 26=0$, the system is not achromatic. To completely remove coupling between $x-x^{\prime}$ and $z-z^{\prime}$ planes, not only the determinant of sub-matrix $R[x z]$ has to be zero, but all of its elements have to be zero. If any element of this sub-matrix is non-zero, it means that beam is not upright in the $\mathrm{x}-\mathrm{z}$ plane. The coupling provided by the space charge will effectively produce dispersion (R16), angular dispersion (R26) and non-zero matrix elements R15 \& R25. The beam ellipse can be made upright in $x-z$ plane by introducing two rf cavities in the achromat.

\section{EXAMPLE}

As shown, all four elements of the $\mathrm{R}$ [xz] sub-matrix should have zero values for a transport line to be achromatic. We will use as an example proposed Spallation Neutron Source (SNS) high energy transport line [3]. This transport line is about 180 meters long and has an achromat which is six cells long, with a total phase advance of 360 degrees. The layout of HEBT is shown in Figure 1, and the corresponding amplitude and dispersion functions are shown in Figure 2.

The transfer matrix of the achromat in the HEBT for the zero current (using TRACE3D) is

$$
R_{\text {acro }}=\left(\begin{array}{llllll}
1 & 0 & 0 & 0 & 0 & 0 \\
0 & 1 & 0 & 0 & 0 & 0 \\
0 & 0 & 1 & -1.305 & 0 & 0 \\
0 & 0 & 0.01778 & 0.98846 & 0 & 0 \\
0 & 0 & 0 & 0 & 1 & 14.15989 \\
0 & 0 & 0 & 0 & 0 & 1
\end{array}\right)
$$

with the following units

$$
\left(\begin{array}{llllll}
1 & m & 1 & m & 1 & m \\
m^{-1} & 1 & m^{-1} & 1 & m^{-1} & 1 \\
1 & m & 1 & m & 1 & m \\
m^{-1} & 1 & m^{-1} & 1 & m^{-1} & 1 \\
1 & m & 1 & m & 1 & m \\
m^{-1} & 1 & m^{-1} & 1 & m^{-1} & 1
\end{array}\right)
$$

The $R[x z]$ sub-matrix of the achromat plus the following two cells, for zero current, is

$$
R[x z]_{\text {acro }+2 c}=\left(\begin{array}{ll}
0 & 0 \\
0 & 0
\end{array}\right)
$$

The $\mathrm{R}[\mathrm{xz}]$ sub-matrix for the achromat, with $28 \mathrm{~mA}$ current, is

$$
R[x z]_{\text {acro }}=\left(\begin{array}{ll}
0.133349 & -0.18495 \\
0.00469 & 0.8130
\end{array}\right)
$$

Now one can apply the conditions $R 16=R 26=0$, by adjusting two families of quadrupoles in the achromat, and the resultant $R[x z]$ sub-matrix of the achromat is

$$
R[x z]_{\text {acro }}=\left(\begin{array}{ll}
0.14278 & 0 \\
0.00339 & 0
\end{array}\right)
$$

The $R[\mathrm{xz}]$ sub-matrix of the achromat plus the following two cells, for the $28 \mathrm{~mA}$, is then

$$
R[x z]_{\text {acrot } 2 c}=\left(\begin{array}{ll}
-0.00608 & -0.68454 \\
-0.01605 & 0.01919
\end{array}\right)
$$

This shows the condition $R 16=R 26=0$, will not yield a achromatic system if one includes space charge. Figure 3 shows the space charge induced dispersion at end of the SNS achromat as function of half bunch length at beginning of the HEBT. 


\section{DISCLAIMER}

This report was prepared as an account of work sponsored by an agency of the United States Government. Neither the United States Government nor any agency thereof, nor any of their employees, makes any warranty, express or implied, or assumes any legal liability or responsibility for the accuracy, completeness, or usefulness of any information, apparatus, product, or process disclosed, or represents that its use would not infringe privately owned rights. Reference herein to any specific commercial product, process, or service by trade name, trademark, manufacturer, or otherwise does not necessarily constitute or imply its endorsement, recommendation, or favoring by the United States Government or any agency thereof. The views and opinions of authors expressed herein do not necessarily state or reflect those of the United States Government or any agency thereof. 


\section{DISCLAIMER}

Portions of this document may be illegible in electronic image products. Images are produced from the best available original document. 


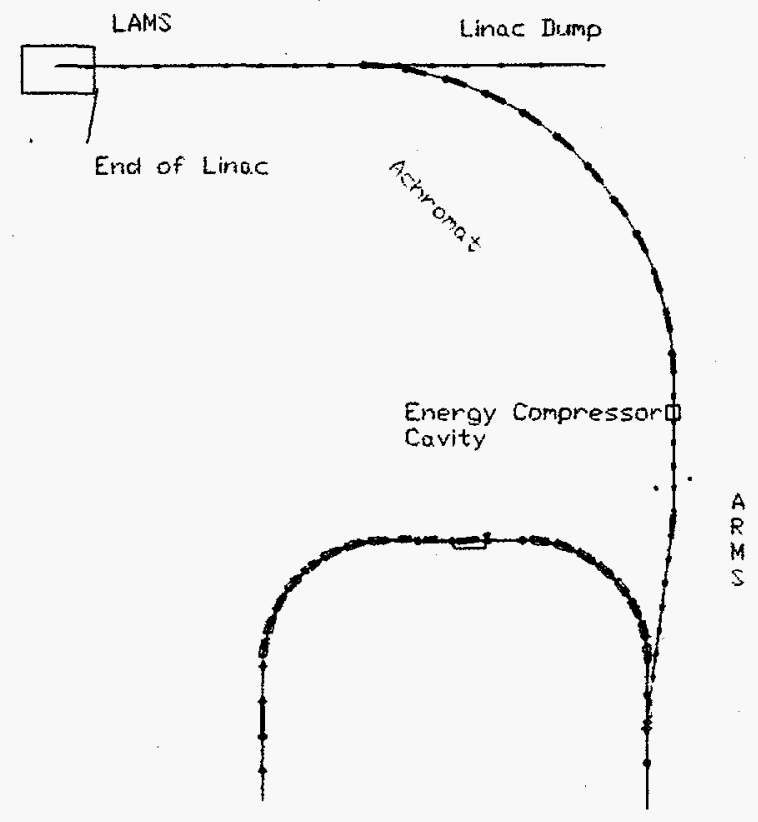

Figure 1: Layout of the SNS HEBT line.

Next, one can apply the conditions $R 16=R 26=R 15$ $=R 25=0$ (instead of $R 16=R 26=0$ ) by adjusting the two families of quadrupoles in the achromat, and adding two small 1 meter RF cavities in the 2nd and 5th cells of the achromat set at appropriate voltages. One can then get the following $R[x z]$ sub-matrix, similar to the zero current case.

$$
R[x z]_{\text {acro }}=\left(\begin{array}{ll}
0 & 0 \\
0 & 0
\end{array}\right)
$$

For this case, however, the $\mathrm{R}[\mathrm{xz}]$ sub-matrix for the achromat plus the following two cells, for $28 \mathrm{~mA}$, is

$$
R[x z]_{\text {acro+2c }}=\left(\begin{array}{ll}
0 & 0 \\
0 & 0
\end{array}\right)
$$

Therefore, for bunched beams achromaticity should be redefined as a line having zero values of matrix elements $\mathrm{R} 15, \mathrm{R} 25, \mathrm{R} 16$ and R26. The additional condition can be achieved with two small if cavities.

\section{REFERENCES}

[1] K. R. Crandall and D. P. Rusthoi, Los Alamos Report LAUR-97-886

[2] P. M. Lapostolle, CERN report AR/nt. SG/65-15, Geneva, Switzerland, July 1965.

[3] D. Raparia, J. Alessi, Y.Y. Lee, W. T. Weng, to be published in Proceedings of 1997 Particle Accelerator Conference, Vancouver, B.C. Canada, May 12-16, 1997.

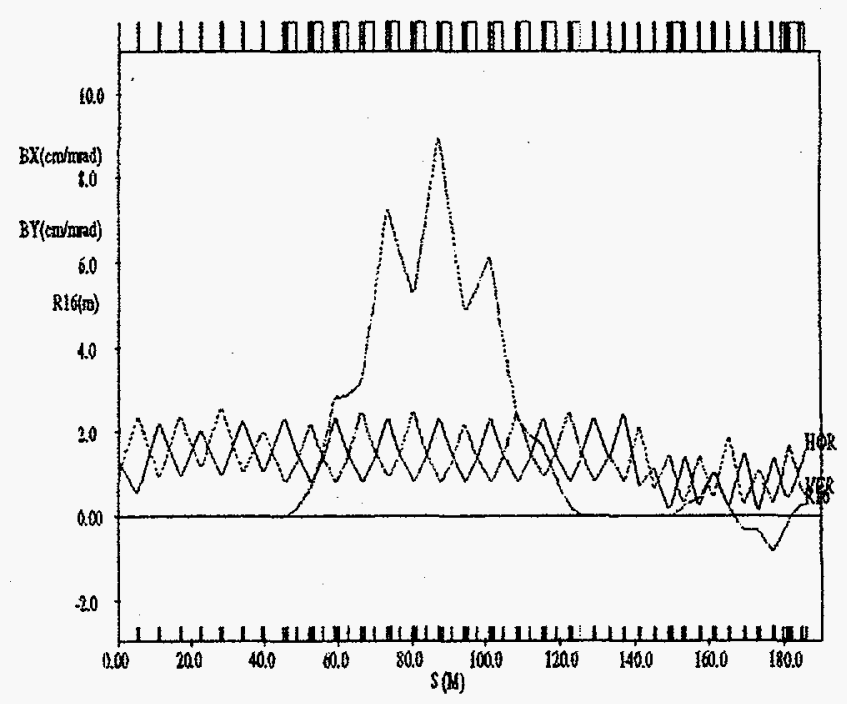

Figure 2: TRANSPORT output for the amplitude $(\beta)$ functions and dispersion function $(\eta)$ along the SNS HEBT.

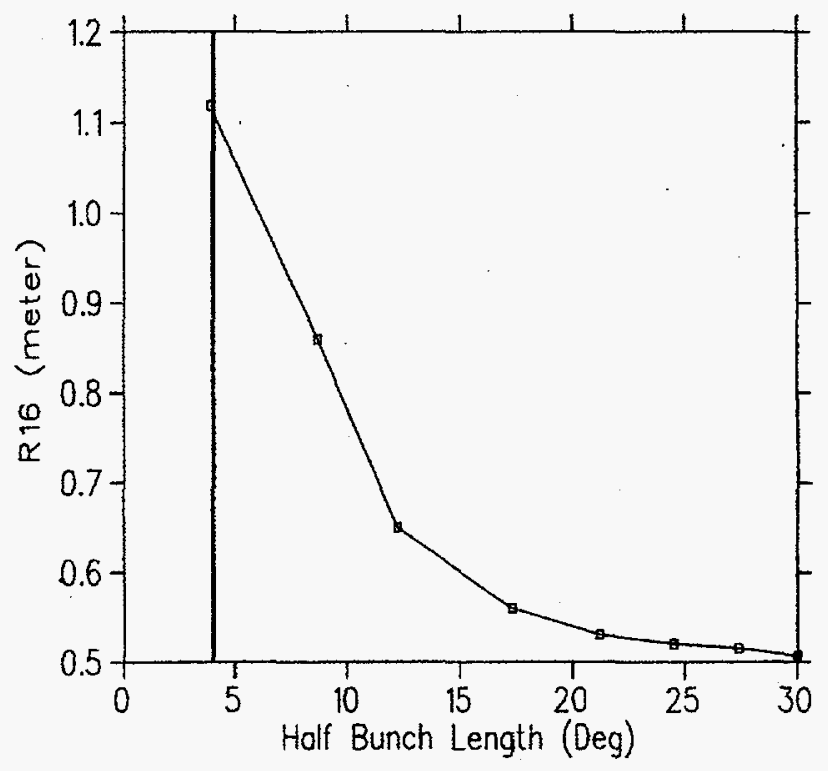

Figure 3: Space-charge induced dispersion (R16) at the end of the SNS achromat as a function of bunch length at the beginning of HEBT. Vertical line shows the design bunch length. 